\title{
Conformally Coupled Scalars, Instantons, and Vacuum Instability in 4D Anti-de Sitter Space
}

\author{
Sebastian de Haro, ${ }^{1}$ Ioannis Papadimitriou, ${ }^{2}$ and Anastasios C. Petkou ${ }^{3}$ \\ ${ }^{1}$ Department of Mathematics, King's College, London WC2R 2LS, United Kingdom \\ ${ }^{2}$ DESY Theory Group, Notkestrasse 85, D-22603 Hamburg, Germany \\ ${ }^{3}$ Department of Physics, University of Crete, 71003 Heraklion, Greece \\ (Received 16 January 2007; published 8 June 2007)
}

\begin{abstract}
We show that a scalar field conformally coupled to AdS gravity in four dimensions with a quartic selfinteraction can be embedded into $M$ theory. The holographic effective potential is exactly calculated, allowing us to study nonperturbatively the stability of $\mathrm{AdS}_{4}$ in the presence of the conformally coupled scalar. It is shown that there exists a one-parameter family of conformal scalar boundary conditions for which the boundary theory has an unstable vacuum. In this case, the bulk theory has instanton solutions that mediate the decay of the $\mathrm{AdS}_{4}$ space. These results match nicely with the vacuum structure and the existence of instantons in an effective three-dimensional boundary model.
\end{abstract}

DOI: 10.1103/PhysRevLett.98.231601

PACS numbers: $11.25 . \mathrm{Tq}, 11.25 . \mathrm{Yb}$

Introduction. - Quantum mechanical tunneling is an important mechanism for vacuum selection in the huge landscape of string theory vacua. It is also expected that it plays a role in the early Universe, and in fact it can become dominant in an eternally inflating universe. In both cases tunneling needs to be understood in the presence of a quantum gravity theory. This is a difficult problem. However, if holography is at work one may hope to map this into a problem of vacuum decay in a theory without gravity which might be more accessible. In any case, it is certainly of interest to study holography in the presence of unstable vacua.

As a step in this direction we study here a simple model that arises as a consistent truncation of $M$ theory to four dimensions. The model consists of a scalar field conformally coupled to gravity with a quartic self-interaction. We calculate the exact holographic effective potential of the dual boundary theory on the sphere and we show that there exists a one-parameter family of boundary conditions for the scalar field such that, in a certain range of this parameter, the boundary theory has an unstable vacuum. In the same parameter range we find instanton solutions whose Lorentzian signature form describes a bubble of true vacuum expanding at the speed of light [1]. However, to understand the end point of the decay it is necessary to go beyond the supergravity approximation and consider finite- $N$ corrections. Finally, we argue that an $O\left(N^{\prime}\right) \phi^{6}$ three-dimensional theory qualitatively reproduces the bulk results.

Conformally coupled scalars and their embedding in string/ $M$ theory. - The action of a scalar field conformally coupled to Einstein gravity with a negative cosmological constant in four dimensions is

$$
S=\frac{1}{2} \int d^{4} x \sqrt{g}\left[\frac{-R+2 \Lambda}{\kappa^{2}}+\left(\partial_{\mu} \phi\right)^{2}+\frac{1}{6} R \phi^{2}+\lambda \phi^{4}\right]
$$

where $\kappa^{2}=8 \pi G_{4}, \lambda$ is a dimensionless coupling, and the cosmological constant is $\Lambda=-3 / l^{2}$. An important property of (1) is that all its extrema have constant Ricci scalar $R=-12 / l^{2}[2]$.

It was pointed out in [3] that, for the special value $\lambda=$ $\kappa^{2} / 6 l^{2}$, (1) can be obtained via the field redefinition

$$
\kappa \phi / \sqrt{6}=\tanh (\kappa \tilde{\phi} / \sqrt{6}), \quad g_{\mu \nu}=\cosh ^{2}(\kappa \tilde{\phi} / \sqrt{6}) \tilde{g}_{\mu \nu},
$$

from an action with a minimally coupled scalar field and the potential $V(\tilde{\phi})=-\left(3 / \kappa^{2} l^{2}\right) \cosh (\sqrt{2} \kappa \tilde{\phi} / \sqrt{3})$. The resulting action is a consistent truncation of the $\mathcal{N}=8$ gauged supergravity action to the diagonal of the Cartan subgroup $U(1)^{4}$ of the $S O(8)$ gauge group [4]. It follows that any solution of (1) with $\lambda=\kappa^{2} / 6 l^{2}$ can be uplifted to a solution of 11-dimensional supergravity. The explicit uplift for this particular one-scalar truncation takes the form [5]:

$$
\begin{aligned}
d \hat{s}_{11}^{2}= & 4\left(X+X^{-1}\right)^{-2} \tilde{\Delta}^{2 / 3} d s_{4}^{2}+4 l^{2} \tilde{\Delta}^{-1 / 3}\left\{X ^ { 3 } \left[\left(\cos ^{2} \theta\right.\right.\right. \\
+ & \left.\left.\left.X^{-4} \sin ^{2} \theta\right) d \theta^{2}+\sin ^{2} \theta d \phi_{4}^{2}\right]+X^{-1} \cos ^{2} \theta d \Omega_{5}^{2}\right\}, \\
\hat{F}_{(4)}= & -16 l^{-1}\left(X+X^{-1}\right)^{-4} \\
& \times\left[2 X^{2} \cos ^{2} \theta+X^{-2}\left(1+2 \sin ^{2} \theta\right)\right] \epsilon_{4} \\
& +16 l \sin 2 \theta\left(X+X^{-1}\right)^{-2} X^{-1} *_{4} d X \wedge d \theta,
\end{aligned}
$$

where we have defined $\tilde{\Delta}=X \cos ^{2} \theta+X^{-3} \sin ^{2} \theta$ and $X=$ $(1+\kappa \phi / \sqrt{6})^{1 / 2} /(1-\kappa \phi / \sqrt{6})^{1 / 2}$. Note that the metric (3) preserves an $S^{5}$ and contains a squashed $S^{2}$, which becomes totally squashed as $\phi \rightarrow \sqrt{6} / \kappa$, or $X \rightarrow \infty$. This signals a breakdown of the supergravity description in this limit.

Boundary conditions and the holographic effective action. - A scalar field in (Euclidean) $\mathrm{AdS}_{4}$ with radius $l$ and in the upper half plane coordinates has the asymptotic 
behavior

$$
\phi \sim z^{\Delta_{-}}\left[\phi_{-}(\vec{z})+\cdots\right]+z^{\Delta_{+}}\left[\phi_{+}(\vec{z})+\cdots\right],
$$

as $z$ approaches the conformal boundary at $z=0$. The parameters $\Delta_{ \pm}$, where $\Delta_{+} \geq \Delta_{-}$, are related to the mass of the scalar by $m^{2} l^{2}=\Delta(\Delta-3)$ and $\phi_{ \pm}(\vec{z})$ are arbitrary functions of the transverse coordinates. It is known that such a scalar can be consistently quantized in $\mathrm{AdS}_{4}$ either with Dirichlet, $\delta \phi_{-}(\vec{z})=0$, or with Neumann, $\delta \phi_{+}(\vec{z})=$ 0 , boundary conditions if the mass squared is in the range $-9 / 4<m^{2} l^{2}<-5 / 4[6]$. This is the case for the scalar $\phi$ in (1) since the scalar curvature $R$ is constant and acts as a mass term with $m^{2} l^{2}=-2$. Quantizing $\phi$ with Neumann boundary conditions one concludes that the dual boundary theory has an operator with dimension $\Delta_{-}=1$ [7]. This is consistent with the $M$ theory embedding discussed above, where the scalar $\tilde{\phi}$ in (2), and more generally all the scalars of the $S L(8, \mathbb{R}) / S O(8)$ submanifold of the scalar manifold of $\mathcal{N}=8$ gauged supergravity, is dual to a dimension one operator whose vacuum expectation value (VEV) parametrizes a certain direction of the Coulomb branch of the $\mathcal{N}=8$ super conformal field theory (SCFT) on the world volume of coincident $M 2$ branes.

In AdS/CFT the (generically nonlocal) relationship between the functions $\phi_{-}$and $\phi_{+}$in the asymptotic expansion (5), which is imposed by the requirement of regularity of the exact solution, determines the effective action of the boundary theory. In particular, the effective action of the dual boundary theory is given by the on-shell value of the renormalized bulk action $S\left[\phi_{-}\right]$, taken as a functional of $\phi_{-}$, by the relation $\Gamma_{\text {eff }}\left[-\phi_{-}\right]=S\left[\phi_{-}\right]$. This follows from the fact that $S\left[\phi_{-}\right]$is minus the generating functional for connected correlation functions of the boundary operator with dimension $\Delta_{+}$and its Legendre transform gives the corresponding generating functional for the boundary operator with dimension $\Delta_{-}$. It follows that the relationship $\delta \Gamma_{\text {eff }}\left[\phi_{-}\right] / \delta \phi_{-}=-\delta S\left[\phi_{-}\right] / \delta \phi_{-}=0$ determines the vacuum structure of the dual boundary theory.

Starting with solutions satisfying Neumann boundary conditions with vanishing source $J(\vec{z})$ for the dual operator, i.e., $\phi_{+}(\vec{z}) \equiv J(\vec{z})=0$, one can modify the boundary conditions while preserving the (bosonic) asymptotic symmetry group of $\mathrm{AdS}_{4}$. There is a one-parameter family of such deformations

$$
\phi_{+}(\vec{z})=-l \alpha \phi_{-}^{2}(\vec{z}) .
$$

These mixed boundary conditions interpolate between Neumann $(\alpha=0)$ and Dirichlet $(\alpha=\infty)$. For generic $\alpha$, the new boundary condition will not be a stationary point of (1) but it can be enforced by adding a boundary term to the action which we will determine. Eventually we will be considering solutions with vanishing stress-energy tensor and for that we renormalize to zero the on-shell contribution of the gravity part of the action. The matter part of the action includes also a generalized Gibbons-Hawking term (see [2] for details). The boundary term that enforces (6) is

$$
S_{\mathrm{bdy}}=-\frac{l^{3} \alpha}{3} \int d^{3} z \phi_{-}^{3}(\vec{z}) \text {. }
$$

In the context of the AdS/CFT correspondence the addition of the boundary term (7) corresponds to a marginal triple-trace deformation of the dual conformal field theory (CFT) [8], completely breaking supersymmetry. The boundary condition (6) has been studied in the context of "designer gravity" [9], where various black hole solutions [8] as well as gravitational solitons and cosmological big bang or crunch geometries [10] satisfying these boundary conditions were numerically constructed. An exact Poincaré domain wall solution satisfying the boundary condition (6) was found and uplifted to 11 dimensions in [5].

The equations of motion following from (1) determine in principle the nonlocal relation between the two modes, $\phi_{ \pm}(\vec{z})$, and hence the holographic effective action for the VEV of the dual dimension one operator in the CFT deformed by the marginal deformation (7). This effective action can be computed in a derivative expansion away from the vanishing VEV point. On a nearly flat boundary one finds that up to two derivatives [2]

$$
\begin{aligned}
\Gamma_{\text {eff }}\left[\phi_{-}\right]= & \frac{1}{6 \sqrt{\lambda}} \int d^{3} x \sqrt{g_{(0)}}\left[\phi_{-}^{-1} \partial_{i} \phi_{-} \partial^{i} \phi_{-}\right. \\
& \left.+\frac{1}{2} R\left[g_{(0)}\right] \phi_{-}+2 \sqrt{\lambda}(\sqrt{\lambda}-\alpha) \phi_{-}^{3}\right],
\end{aligned}
$$

where $g_{(0) i j}$ is the boundary metric. Moreover, the exact holographic effective potential for $R \geq 0$ is [2]

$$
V_{\lambda, \alpha}\left(\phi_{-}\right)=\frac{1}{3 \lambda}\left[\left(\frac{R}{6}+\lambda \phi_{-}^{2}\right)^{3 / 2}-\alpha \lambda \phi_{-}^{3}-\left(\frac{R}{6}\right)^{3 / 2}\right],
$$

where the additive constant has been fixed by requiring that the trivial vacuum at $\phi_{-}=0$ has zero energy. Redefining $\phi_{-}=\varphi^{2}$ and taking $\alpha \rightarrow \sqrt{\lambda}$ the two-derivative holographic effective action (8) takes the form

$$
\begin{aligned}
\Gamma_{\mathrm{eff}}[\varphi]= & \frac{4}{3 \sqrt{\lambda}} \int d^{3} x \sqrt{g_{(0)}}\left(\frac{1}{2} \partial_{i} \varphi \partial^{i} \varphi+\frac{1}{16} R\left[g_{(0)}\right] \varphi^{2}\right. \\
& \left.+\frac{1}{8} \mu \varphi^{6}\right)+\mathcal{O}\left(\mu^{2}\right),
\end{aligned}
$$

where $\mu \equiv \lambda-\alpha^{2}$. This agrees with the classically conformally invariant toy-model actions used in $[10,11]$.

Since the system (1) can be embedded into 11dimensional supergravity, the holographically dual field theory is (a sector of) the $\mathcal{N}=8$ interacting SCFT in the large- $N$ limit [12]. In the Abelian case, $N=1$, this theory can be obtained by compactifying $\mathcal{N}=4$ super Yang-Mills in four dimensions on a circle in the limit of zero radius, and it is also believed to be the infrared fixed point of $\mathcal{N}=8$ super Yang-Mills in three dimensions. 
However, little is known about this theory, which makes any test of the AdS/CFT correspondence very difficult. Nevertheless, we will argue below that the bulk results (8)(10) can be qualitatively reproduced by a certain threedimensional $O\left(N^{\prime}\right)$ model, where $N^{\prime}$ is not related to the number of $M 2$ branes. This not only implies some connection between the $\mathcal{N}=8$ SCFT and the $O\left(N^{\prime}\right)$ model, as a consequence of the AdS/CFT correspondence, but it is also a step towards finding the holographic dual of $O\left(N^{\prime}\right)$ models in three dimensions [13].

Scalar instantons:-The equations of motion following from the action (1) admit nontrivial solutions with vanishing stress tensor. The condition that the stress tensor vanishes reduces to a linear equation for the scalar field, namely $\left(\nabla_{\mu} \nabla_{\nu}-\frac{1}{4} g_{\mu \nu} \square_{g}\right) \phi^{-1}=0$ [2], which admits nontrivial solutions provided the metric is that of exact $\mathrm{AdS}_{4}$. In the upper half plane coordinates the most general solution of the equations of motion with vanishing bulk stress tensor takes the form

$$
\phi=\frac{2}{l \sqrt{|\lambda|}}\left(\frac{b z}{-\operatorname{sgn}(\lambda) b^{2}+(z+a)^{2}+\left(\vec{z}-\vec{z}_{0}\right)^{2}}\right),
$$

where $a, b, z_{0}^{i}, i=1,2,3$, are arbitrary constants. For $\lambda>0$, this Euclidean solution is nonsingular provided

$$
a>b \geq 0 .
$$

For $\lambda<0$ this solution was studied in [11] ignoring its backreaction on the geometry. We have now shown that in fact there is no backreaction and (11) together with the $\mathrm{AdS}_{4}$ metric is an exact solution of the coupled equations of motion. Expanding the solution (11) asymptotically near the conformal boundary we get precisely the expansion (5), where $\phi_{ \pm}$satisfy (6) with $\alpha \equiv \sqrt{|\lambda|} a / b$ and

$$
\phi_{-}=\frac{2}{l \sqrt{|\lambda|}}\left(\frac{b}{-\operatorname{sgn}(\lambda) b^{2}+a^{2}+\left(\vec{z}-\vec{z}_{0}\right)^{2}}\right) .
$$

Notice that the parameter $\alpha$ is not a modulus of the solution but rather labels different boundary conditions. Therefore, it corresponds to the deformation parameter of the dual theory and different values of $\alpha$ correspond to saddle points of different theories. The remaining parameters in (11) do correspond to moduli of the solution and they parametrize a four-dimensional hyperbolic space $\tilde{\dddot{H}}_{4}$ of radius $\tilde{l}=\sqrt{\alpha^{2}-\lambda}$, which is also the moduli space of instantons in $\mathbb{R}^{3}$. This can be seen by parametrizing the solution (11) in a manifestly $O(5,1)$ covariant form as in [14], $\phi^{-1}=h_{M} y^{M}$, where $h, y$ are vectors in a sixdimensional embedding space. It can then be shown [2] that the solution is determined by the five-dimensional part of $h$, which satisfies $h^{2}=-\frac{|\lambda|}{b^{2}}\left[a^{2}-b^{2} \operatorname{sgn}(\lambda)\right]$ and hence, given (12), it parametrizes an $\tilde{\mathbb{U}}_{4}$ also for $\lambda>0$. Note that the condition (12) for the solution to be nonsingular is the necessary and sufficient condition for the radius of the moduli space to be well defined and also for the potential
(9) to be unbounded from below. Remarkably, the VEV (13) is an exact extremum of the two-derivative holographic effective action (10).

Vacuum decay. - The unboundedness of the boundary effective potential for $\alpha>\kappa / \sqrt{6} l$ is the holographic image of an $\mathrm{AdS}_{4}$ instability towards its spontaneous "dressing" by a nonzero scalar field when the boundary condition (6) is imposed with $\alpha>\kappa / \sqrt{6} l$. This nonperturbative instability does not contradict any positivity theorem. Although the positivity theorems $[6,15-17]$ do apply when the supersymmetric $(\alpha=\kappa / \sqrt{6} l[2])$ boundary conditions are imposed on the scalar field, they do not apply for a generic value of the deformation parameter $\alpha$ in (7). The question of stability of AdS with such boundary conditions has been addressed in the context of "designer gravity" $[9,18]$, where it has been suggested that AdS with boundary conditions on the scalar defined by the boundary term $S_{\mathcal{W}}=$ $-\int d^{3} z \mathcal{W}\left(\phi_{-}\right)$is stable provided the function $\mathcal{W}\left(\phi_{-}\right)$ has a global minimum. This is clearly not the case for the deformation (7) and so these positivity theorems do not apply either.

The physical meaning of the instanton solutions (11) becomes clear by taking the boundary to be $S^{3}$. In this case the potential (9) has a global minimum at $\phi_{-}=0$ for $\alpha \leq$ $\sqrt{\lambda}$ (note that $\phi_{-} \geq 0$ ) which turns into a local minimum for $\alpha>\sqrt{\lambda}$ separated by a potential barrier from the instability region at $\phi_{-} \rightarrow \infty$ (Fig. 1). The scalar field can tunnel from the local minimum at $\phi_{-}=0$ to the instability region. In terms of the bulk scalar field the instability region is reached for $\phi \rightarrow \sqrt{6} / \kappa$ or $\tilde{\phi} \rightarrow \pm \infty$. We have seen that this is precisely the limit where an $S^{2}$ in the uplifted 11-dimensional metric (3) gets totally squashed giving rise to a singularity. To understand the true vacuum of the theory for $\alpha>\kappa / \sqrt{6} l$, if there is one, one needs to go beyond supergravity to include finite- $N$ corrections. This would modify the holographic potential (9) and at the same time would resolve the geometric singularity from the squashed $S^{2}$.

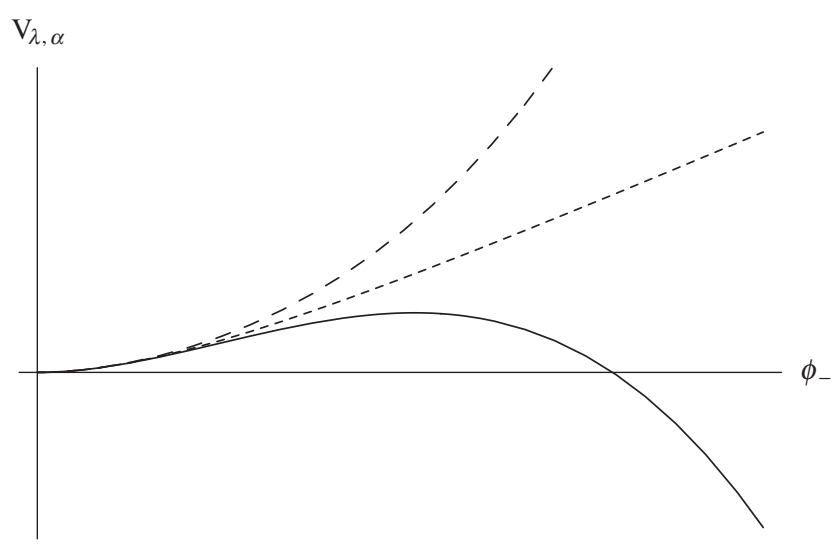

FIG. 1. Plot of the potential (9) on $S^{3}$ for $\alpha<\kappa / \sqrt{6} l$ (long dashes), $\alpha=\kappa / \sqrt{6} l$ (short dashes), and $\alpha>\kappa / \sqrt{6} l$. 
The physical picture of the decay process can be understood in terms of bubble nucleation as proposed by Coleman and De Luccia [1]. Namely, the Lorentzian version of the instanton solution (11) describes a "half bubble" centered on the boundary which is expanding or collapsing at the speed of light towards the bulk. The equatorial plane of the bubble describes an expanding or collapsing bubble in the dual field theory. Outside and far away from the bubble the space is $\mathrm{AdS}_{4}$ corresponding to the local minimum at $\phi_{-}=0$ of the potential (9). Inside the bubble is the "true vacuum" which cannot be understood in the supergravity approximation. The tunneling probability is proportional to the exponential of the onshell action computed with the boundary conditions defined by (7) and evaluated on the instanton solution (11), $\mathcal{P} \propto \exp \left(-\left.\Gamma_{\text {eff }}\right|_{\text {inst }}\right)$. Evaluating this gives [2]

$$
\Gamma_{\text {eff }} \mathrm{l}_{\text {inst }}=\frac{4 \pi^{2} l^{2}}{\kappa^{2}}\left(\frac{1}{\sqrt{1-\kappa^{2} / 6 l^{2} \alpha^{2}}}-1\right) .
$$

Note that the deformation parameter $\alpha$ drives the theory from the regime of marginal stability at $\alpha=\kappa / \sqrt{6} l$ to total instability at $\alpha \rightarrow \infty$. In the global coordinates of $\mathbb{H}_{4}$ where the boundary is $S^{3}$, the instanton solution (11) depends only on the $\mathbb{H}_{4}$ radius. In particular, $\phi_{-}$is constant in these coordinates. This positive constant solution corresponds precisely to the local maximum of the holographic potential (9) on $S^{3}$, which again only exists for $\alpha>\kappa / \sqrt{6} l$. Evaluating the potential at this maximum and multiplying with the volume of $S^{3}$ we get exactly (14). This confirms that the tunneling probability is indeed proportional to the exponential of minus the height of the potential barrier and justifies our claim that the instantons mediate the tunneling of the local minimum at $\phi_{-}=0$ to the instability region for $\alpha>\kappa / \sqrt{6} l$.

An effective boundary theory. - There is some indication that the holographic results (9) and (10) could be reproduced from an $O\left(N^{\prime}\right)$ model in three dimensions with a $g\left(\vec{\phi}^{2}\right)^{3} / 6$ interaction. Both the form (10) of the holographic effective action in the double scaling limit and the existence of instantons in certain $O\left(N^{\prime}\right)$ models in three dimensions are suggestive of such a connection.

To illustrate this point we consider the action

$$
I^{g}=\mathcal{C} \int d^{3} \vec{x}\left[\frac{1}{2} \partial_{i} \phi^{a} \partial^{i} \phi^{a}+\frac{g}{6 N^{\prime 2}}\left(\phi^{a} \phi^{a}\right)^{3}\right],
$$

where $a=1,2, \ldots, N^{\prime}$ and $\mathcal{C}$ is an $N^{\prime}$-independent constant. For $g<0$ this model has instanton configurations given by

$$
\phi^{a}(\vec{x})=\left[3 N^{\prime 2} /(-g)\right]^{1 / 4}\left(c^{a} / \sqrt{b}\right)\left[b^{2}+\left(\vec{x}-\vec{x}_{0}\right)^{2}\right]^{-1 / 2},
$$

where $c^{a} c^{a}=b^{2}$ and $b$ is an arbitrary constant. On $S^{3}$ the classical potential for the $O\left(N^{\prime}\right)$-singlet operator $\sigma \equiv$ $\phi^{a} \phi^{a} / N^{\prime}$ becomes $V_{g}(\sigma)=\mathcal{C}\left(R \sigma / 16+g \sigma^{3} / 6\right)$. With the identifications $\mathcal{C}=4 / 3 \sqrt{\lambda}, \quad g=3 \sqrt{\lambda}(\sqrt{\lambda}-\alpha) / 2$, and $\sigma=\phi_{-}$, this potential coincides with the holographic potential (9) in the limit of small curvature. In particular, the instability region of (9) for $\alpha>\sqrt{\lambda}$ is mapped precisely to the instability region of the $O\left(N^{\prime}\right)$ model for $g<$ 0 . It would be very interesting if a full quantum treatment of this $O\left(N^{\prime}\right)$ model reproduced the full holographic potential (9) in the large- $N^{\prime}$ limit [19]. Evaluating the action (15) on the instanton solution (16) we obtain $N^{\prime-1} I_{\text {inst }}^{g}=$ $\left(\sqrt{2} \pi^{2} / 3 \lambda\right)(\alpha / \sqrt{\lambda}-1)^{-1 / 2}$, which precisely agrees with the tunneling probability (14) in the approximation $\alpha / \sqrt{\lambda} \approx 1$.

I. P. thanks the Aspen Center for Physics for hospitality during the workshop on "Recent Advances in Black Hole Physics in String Theory," as well as the Albert Einstein Institute at Potsdam and the University of Crete, where part of this work was done. A. C. P. wishes to thank T. Tomaras for very useful discussions. A. C.P. is partially supported by the European RTN "Superstrings" and the Greek Research Program "PYTHAGORAS II".

[1] S. R. Coleman and F. De Luccia, Phys. Rev. D 21, 3305 (1980).

[2] I. Papadimitriou, arXiv:hep-th/0703152.

[3] C. Martinez, R. Troncoso, and J. Zanelli, Phys. Rev. D 70, 084035 (2004).

[4] M. J. Duff and J. T. Liu, Nucl. Phys. B554, 237 (1999).

[5] I. Papadimitriou, J. High Energy Phys. 02 (2007) 008.

[6] P. Breitenlohner and D.Z. Freedman, Ann. Phys. (Berlin) 144, 249 (1982).

[7] I. R. Klebanov and E. Witten, Nucl. Phys. B556, 89 (1999).

[8] T. Hertog and K. Maeda, J. High Energy Phys. 07 (2004) 051.

[9] T. Hertog and G. T. Horowitz, Phys. Rev. Lett. 94, 221301 (2005).

[10] T. Hertog and G. T. Horowitz, J. High Energy Phys. 07 (2004) 073.

[11] S. de Haro and A. C. Petkou, J. High Energy Phys. 12 (2006) 076.

[12] N. Seiberg, Nucl. Phys. B, Proc. Suppl. 67, 158 (1998).

[13] I. R. Klebanov and A. M. Polyakov, Phys. Lett. B 550, 213 (2002).

[14] S. Fubini, Nuovo Cimento Soc. Ital. Fis. A 34, 521 (1976).

[15] P. Breitenlohner and D.Z. Freedman, Phys. Lett. 115B, 197 (1982).

[16] G. W. Gibbons, C. M. Hull, and N. P. Warner, Nucl. Phys. B218, 173 (1983).

[17] P. K. Townsend, Phys. Lett. 148B, 55 (1984).

[18] T. Hertog and S. Hollands, Classical Quantum Gravity 22, 5323 (2005).

[19] Work in progress. 